\title{
Autoregulation of the splicing of transcripts from the transformer-2 gene of Drosophila
}

\author{
William Mattox ${ }^{1}$ and Bruce S. Baker \\ Department of Biological Sciences, Stanford University, Stanford, California 94305 USA
}

The Drosophila transformer-2 gene uses alternative promoters and splicing patterns to generate four different mRNAs that together encode three putative RNA-binding polypeptides. The transformer-2 products expressed in somatic tissues function to regulate the RNA splicing of the sex determination gene doublesex, whereas products expressed in the male germ line play an unknown, but essential, role in spermatogenesis. Two alternatively spliced transformer-2 transcripts, each encoding a different putative RNA-binding protein, are found only in the male germ line. These male germ line-specific mRNAs differ from each other by the presence or absence of a single intron called M1. We show that M1-containing transcripts make up a majority of transformer-2 germ-line transcripts in wild-type males but fail to accumulate in males homozygous for transformer-2 null mutations. Germ-line transformation experiments using a variety of reporter gene constructs demonstrate that specific polypeptide products of the transformer-2 gene itself normally repress M1 splicing in the male germ line. Thus, in addition to its role in the sex-specific control of doublesex RNA splicing in somatic tissues, the transformer-2 gene also regulates the splicing of its own transcripts in the male germ line. We propose that this autoregulatory function may serve in negative feedback control of transformer-2 activity during spermatogenesis. The finding that transformer-2 controls multiple splicing decisions suggests that a variety of different alternative splicing choices could be regulated by a relatively limited number of trans-acting factors.

[Key Words: Male germ line; intron retention; sex determination]

Received January 15, 1991; revised version accepted February 21, 1991.

\begin{abstract}
Although many alternative splicing events that control gene expression have been described (for review, see Smith et al. 1989), little is known about the trans-acting regulatory factors that control splicing patterns. The Drosophila transformer-2 (tra-2) gene is one of a small, but growing, number of regulatory genes that have been found to play key roles in the developmentally specific control of alternative RNA splicing reactions. The tra-2 gene functions to control the sex-specific splicing of transcripts from the doublesex $(d s x)$ gene in somatic tissues (Nagoshi et al. 1988). Regulation of the $d s x$ RNA splicing pattern by tra-2 is essential for normal sexual development, as the sex-specific products that are encoded by the alternatively spliced male- and female-specific $d s x$ mRNAs function in a wide variety of somatic tissues to direct sexual differentiation (Baker and Ridge 1980; Baker and Wolfner 1988; Baker 1989; Burtis and Baker 1989).

Molecular analysis of the tra-2 gene has revealed that two alternatively spliced tra-2 mRNAs are expressed in somatic tissues and that each encodes a different polypeptide (Amrein et al. 1990; Mattox et al. 1990). Al-

\footnotetext{
${ }^{1}$ Corresponding author.
}

though the mechanism by which these tra-2 products regulate $d s x$ RNA splicing is unknown, each of the encoded tra-2 polypeptides contains an 80 -amino-acid putative RNA-binding domain that is similar to sequences found in a number of known and putative RNA-binding proteins (Amrein et al. 1988, 1989; Goralski et al. 1989). This domain consists of a well-conserved 8-amino-acid segment known as the RNP consensus sequence (RNPCS), as well as a number of other conserved residues that are spread over the 80-amino-acid region. Among the other proteins that have been identified with this RNAbinding domain are many that carry out general functions during RNA processing (for reviews, see Dreyfuss et al. 1988; Mattaj 1989) and at least one protein involved in developmentally specific regulation of alternative splicing patterns (Bell et al. 1988). In those RNAbinding proteins where the minimal region required for sequence-specific binding to RNA has been determined, the regions identified include the conserved domain and a small amount of flanking sequence (Sachs et al. 1986; Query et al. 1989; Scherly et al. 1989). These and other studies (Merrill 1988; Scherly et al. 1990) suggest that this domain forms an RNA-binding site. The presence of an RNA-binding domain within somatically expressed 
tra-2 polypeptides suggests that these polypeptides participate directly in the $d s x$ RNA splicing reaction by binding to $d s x$ pre-mRNA.

Although it is clear that the tra-2 gene functions in somatic tissues to regulate sexual differentiation (Baker and Ridge 1980; Ota et al. 1981; Belote and Baker 1982, 1987; Belote et al. 1985), the role of the tra-2 products in germ-line tissues, where they are most abundantly expressed, is less well defined. In the male germ line, it is known that $t r a-2$ performs an essential function during a discrete period in spermatogenesis near meiosis (Belote and Baker 1983). Chromosomal males mutant for tra-2 are sterile and produce morphologically abnormal sperm.

Molecular analysis of tra-2 expression in male germ cells has revealed that two tra-2 transcripts are unique to this tissue (Amrein et al. 1990; Mattox et al. 1990). These male germ line-specific transcripts differ from each other by their splicing patterns, and, as with their somatic counterparts, each encodes a polypeptide containing a putative RNA-binding domain. The presence of the RNA-binding domain within these polypeptides suggests that the tra-2 products expressed in the male germ line might also play a role in some aspect of RNA processing or stability. Here, we report results from germline transformation studies that support this idea. These results show that tra-2 products function to autoregulate the alternative splicing of male germ line-specific tra-2 transcripts.

\section{Results}

A nonsense mutation within the tra-2 gene affects the accumulation of male germ line-specific tra-2 transcripts

Due to the use of both alternative splicing pathways and alternative transcription start sites, a variety of different mRNAs are expressed from the tra-2 gene (Amrein et al. 1990; Mattox et al. 1990). The four predominant types of polyadenylated transcripts expressed in adult flies are shown in Figure 1. Type A and B mRNAs are transcribed from the upstream tra-2 promoter. These mRNAs encode polypeptides of 264 and 226 amino acids, respectively, each of which contains a putative RNA-binding domain. These mRNAs are found in the soma of both sexes and in the female germ line. Type $\mathrm{C}$ and $\mathrm{E}$ mRNAs are transcribed from the downstream tra-2 promoter, which is active only in the male germ line. Transcription of both of the male germ line-specific mRNAs initiates within exon 3. These transcripts differ from each other by the presence or absence of the M1 intron located between exons 3 and 4 . Type $E$ transcripts lack the $M 1$ sequences, whereas type $C$ transcripts contain these sequences. Type $C$ transcripts are predicted to encode a polypeptide of 179 amino acids that includes the RNAbinding domain and initiates at an AUG downstream from the $M 1$ intron. Splicing of the $M 1$ intron, as in type E transcripts, forms an AUG at the splice junction. Translation of type E transcripts initiating at this codon is expected to produce a 226-amino-acid polypeptide identical to that encoded by type B mRNA.

Splicing of the M1 intron is much less efficient than splicing of other tra-2 introns (Amrein et al. 1990; Mattox et al. 1990|, such that M1-containing transcripts make up $60-70 \%$ of the polyadenylated tra-2 RNA present in the adult male germ line. In contrast, no polyadenylated transcripts containing other tra-2 introns are detected in adult RNA.

The first indication that mutations in the tra-2 gene affect the splicing of its RNA came from an analysis of tra-2 transcripts present in male individuals homozygous for $\operatorname{tr} a-2^{B}$, a null point mutation. We found that type $C$ transcripts are absent in these individuals. This effect can be seen in the nuclease protection experiment shown in Figure 2. A protected fragment indicative of type C RNA is detected using poly $(A)^{+}$RNA from Canton-S males (Fig. 2, lane 5) but not when poly $(\mathrm{A})^{+}$RNA from tra $-2^{B} /$ tra $-2^{B}$ males is used (Fig. 2, lane 6). The absence of type $C$ transcripts in tra- $2^{B}$ males is accompanied by a two- to threefold increase (as measured by densitometry) in the amount of type $\mathrm{E}$ transcripts present (Fig. 2, cf. lanes 5 and 6). This increase is about the
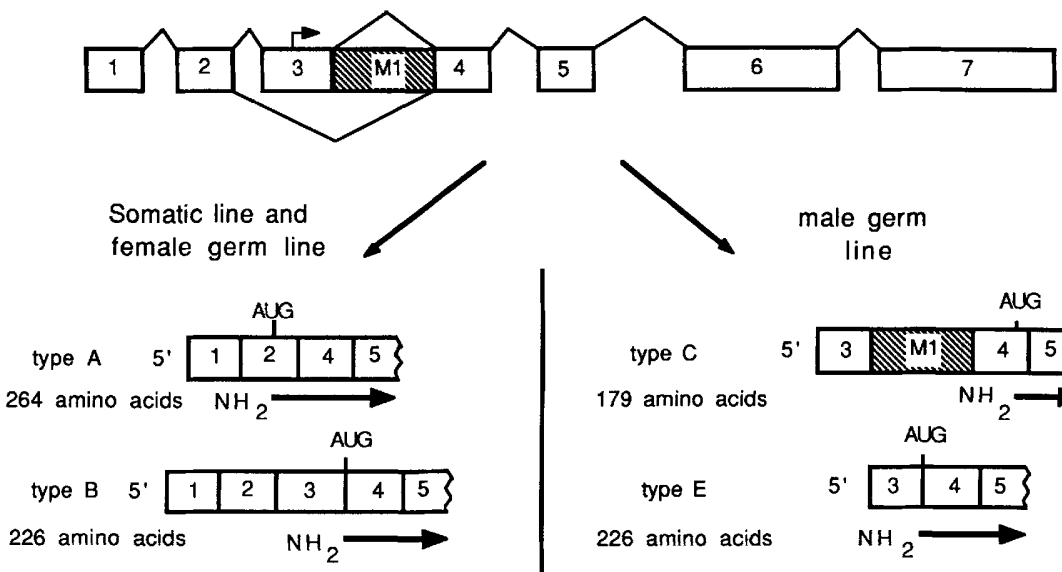

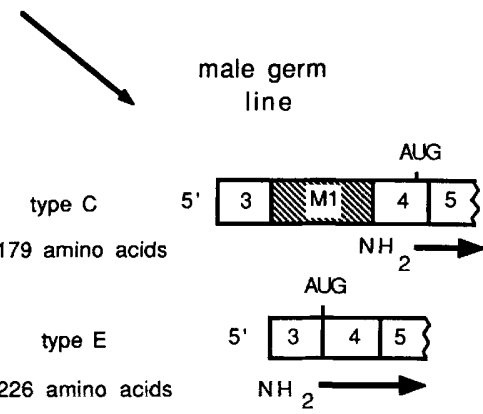

226 amino acids
Figure 1. Alternative tra-2 transcripts. The organization of the tra-2 gene is shown at the top. Exons are indicated by open boxes. The M1 intron is indicated by a hatched box. Other introns are indicated by the blank regions between exons. The position of the male germ line-specific transcription start site is indicated by an arrow over exon 3 . Shown below the gene are the differentially processed portions of each of the major tra-2 mRNAs. Exons 5-7 are also present in each of these RNAs. Alternative splicing is predicted to result in translation of products initiating at three different AUG codons. The position of the predicted initiation codon in each mRNA is indicated along with the size of the expected polypeptide. Type B and E RNAs are expected to use the same initiation codon. This codon is formed in the splicing of exons 3 and 4 . 


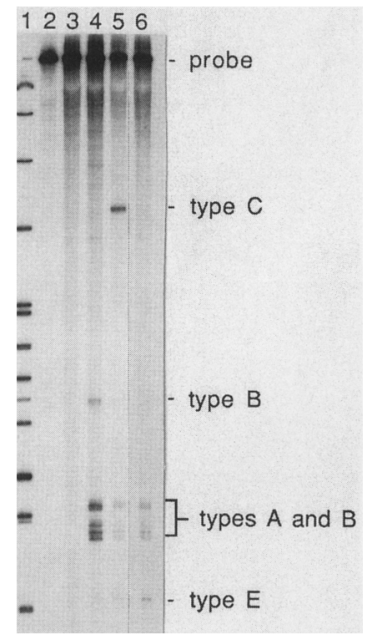

Figure 2. Mung bean nuclease protection analysis of transcripts from $t r a-2^{B}$ males. Protection of a uniformly labeled single-stranded DNA probe by various poly $(A)^{+}$RNA samples is shown. The probe used derives from tra-2 genomic sequences and extends over the region between the middle of the Ml intron and the $5^{\prime}$ end of exon 1. Protected fragments that are indicative of the various types of tra-2 transcripts are indicated at the right of the gel. Because the probe is labeled uniformly the intensity of bands is a product of both the quantity of the hybridizing RNA and the size of the protected fragment. (Lane 1) A pBR322 HpaII digest as size standards; (lane 2) probe treated without nuclease; (lanes 3-6) samples treated with nuclease after hybridization with either no RNA (lane 3), wild-type (Canton-S) female RNA (lane 4), wild-type male RNA (lane 5), or tra $-2^{B}$ male RNA (lane 6).

amount expected if all type $C$ transcripts normally present are spliced to form type $\mathrm{E}$ transcripts in $t r a-2^{B}$ males. No effects on the levels of type A and B transcripts were observed.

We also found type $C$ transcripts to be absent from males that were either homozygous (not shown) or hemizygous for the original tra-2 allele, referred to here as tra- $2^{1}$, which was isolated by Watanabe (1975). When poly $(\mathbf{A})^{+}$RNAs from wild-type females and males was hybridized with a probe derived exclusively from the M1 intron we detected a male-specific $1.6-\mathrm{kb}$ transcript, as expected for type C RNA (Fig. 3, lanes 1 and 2, top). No similar band was detected in poly $(\mathrm{A})^{+}$RNA from $t r a-2^{1}$ males (Fig. 3, lane 4, top) demonstrating that, like tra- $2^{B}$, this mutation affects the accumulation of type $\mathrm{C}$ transcripts. Hybridization of the same blot with a probe homologous to all four of the major tra-2 transcripts (Fig. 3, lanes 1-4, middle/ resulted in detection of a single band migrating at $\sim 1.5-1.6 \mathrm{~kb}$ in both the mutant and in the wild-type RNA samples. Previous studies (Goralski et al. 1989; Mattox et al. 1990) have demonstrated that due to the similarity in their sizes, the various alternative tra-2 RNAs are not well resolved on formaldehyde gels. Thus, this band represents the sum of hybridization to types A, $B, C$, and $E$ transcripts. The relative intensity of this band in RNA from wild-type and mutant individuals suggests that the overall level of tra-2 RNA (the sum of types $\mathrm{A}, \mathrm{B}, \mathrm{C}$, and $\mathrm{E}$ ) is not affected by the tra- $2^{1}$ mutation. Attempts to examine the effects of the tra- $2^{1} \mathrm{mu}$ tation on type A, B, and E transcripts individually by nuclease protection analysis were complicated by the presence of a commonly found sequence polymorphism (Amrein et al. 1990) within exon 3 of the tra-2 ${ }^{1}$ gene (data not shown). Despite this limitation, the above blot hybridization results show that the tra- $2^{1}$ mutation, as with $\operatorname{tra}-2^{B}$, has a strong specific effect on the accumulation of type C RNA.

The absence of type $C$ transcripts in $t r a-2^{B}$ and $t r a-2^{1}$ males might be explained in either of two ways. (1) These mutations each affect cis-acting regulatory sequences located within or near the Ml intron that are required for repression of $\mathrm{Ml}$ splicing or for the stability of transcripts containing the M1 intron. (2) These mutations each affect the function of a product of the tra-2 gene itself that normally acts to repress M1 splicing or to stabilize Ml-containing transcripts.

To investigate the possibility that $t r a-2^{B}$ affects cisacting regulatory sequences, we determined the sequence of the region of the $t r a-2^{B}$ gene that is located between the male germ line-specific transcription start site in exon 3 and the $3^{\prime}$ end of the protein-coding sequences in exon 7 (for details, see Materials and methods). No sequence differences were found within the M1 intron or in the exons that immediately flank it. A single $\mathrm{C}$ to $\mathrm{T}$ transition was found, however, within exon 5 (Fig. 4). This mutation creates an in-frame nonsense codon that is expected to cause truncation of the polypeptides encoded by each of the major tra-2 transcripts at a position that is within the putative RNA-binding domain and 155 amino acids upstream from their shared carboxyl terminus. The fact that the nucleotide altered in

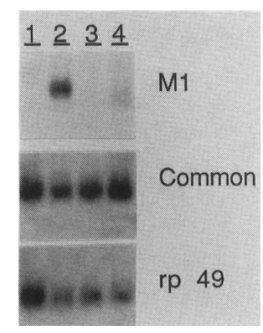

Figure 3. Expression of type $C$ transcripts in $t r a-2^{B}$ and $t r a-2^{1}$ males. Poly $(\mathrm{A})^{+}$RNAs were hybridized with the indicated probes after electrophoresis and transfer to membranes. To eliminate the remote possiblity of effects from extragenic recessive modifiers each allele was tested over a deficiency for the tra-2 gene $[D f(2 R)$ Trix $]$ rather than as a homozygote. The lanes were loaded with equal amounts of RNA from $+/ D f(2 R)$ Trix females (lane 1), + IDf(2R)Trix males (lane 2), tra- $2^{B} / D f(2 R)$ Trix males (lane 3), and tra- $2^{1} / D f(2 R)$ Trix males (lane 4). The top panel was hybridized with a probe containing the first $202 \mathrm{nu}-$ cleotides of the Ml intron, the middle panel with a probe spanning exons 4 and 5 , and the bottom panel was hybridized with a probe from the Drosophila ribosomal protein 49 gene (rp49) (O'Connell and Rosbash 1984). Hybridization to rp49 RNA was used as a control for consistent loading of the gel. 


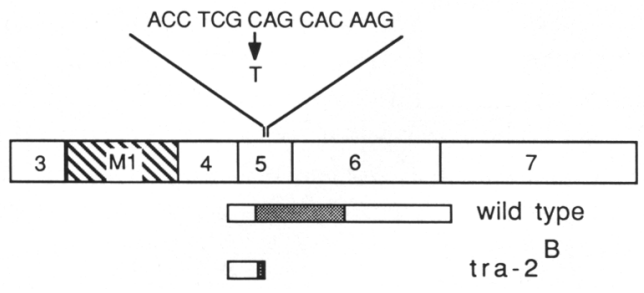

Figure 4. The $t r a-2^{B}$ gene contains a nonsense mutation in exon 5 . The sequence immediately surrounding the nonsense mutation in $\operatorname{tra}-2^{B}$ is shown as well as its position within exon 5. The mRNA shown is spliced in the type $\mathrm{C}$ pattern. Below it the predicted wild-type and $t r a-2^{B}$ translation products are shown. The positions of the putative RNA-binding domain within tra-2 polypeptides are indicated by the shaded regions. Translation products from other types of tra-2 transcripts would be similarly truncated.

the $\operatorname{tra}-2^{B}$ allele is located in a region that is shared by all tra-2 transcripts and is somewhat distant from the M1 intron argues against the idea that this mutation affects cis-acting regulatory sequences that are specifically involved in the processing or stability of M1-containing transcripts. This led us to examine the second of the two hypotheses offered above: that tra-2 products play a role in the accumulation of M1-containing transcripts.

\section{Expression of type C RNA is dependent on a trans-acting function of the tra-2 gene}

To test the idea that tra-2 products are normally required for the accumulation of type $C$ transcripts, we asked whether a functional tra-2 gene can act in trans to regulate the accumulation of transcripts containing the Ml intron from a tra-2/lac $Z$ reporter gene. The reporter gene used was designed to express $\beta$-galactosidase activity only when the Ml intron is retained. This reporter gene is transcribed from the tra-2 promoter and is contained in the plasmid pCZPORF3 (Fig. 5A). It contains a 7-nucleotide insertion within exon 4 that disrupts the open reading frames (ORFs) encoded by type A, B, and $E$ RNAs. The ORF encoded by type C RNA initiates at an AUG downstream of this insertion, near the $3^{\prime}$ end of exon 4 and, therefore, should not be affected by this frameshift mutation. A portion of the Escherichia coli lac $Z$ gene, sufficient to encode an enzymatically active product, is fused to tra-2-coding sequences downstream to and in-frame with this initiation codon. Transcripts from this fusion gene that are spliced like type A, B, like and $E$ mRNAs are expected to be translated into frameshifted products that terminate before reaching the $l a c Z$ coding sequences. Transcripts retaining the $M 1$ intron are expected to be translated into a fusion polypeptide containing 52 residues from the normal amino terminus of the

A
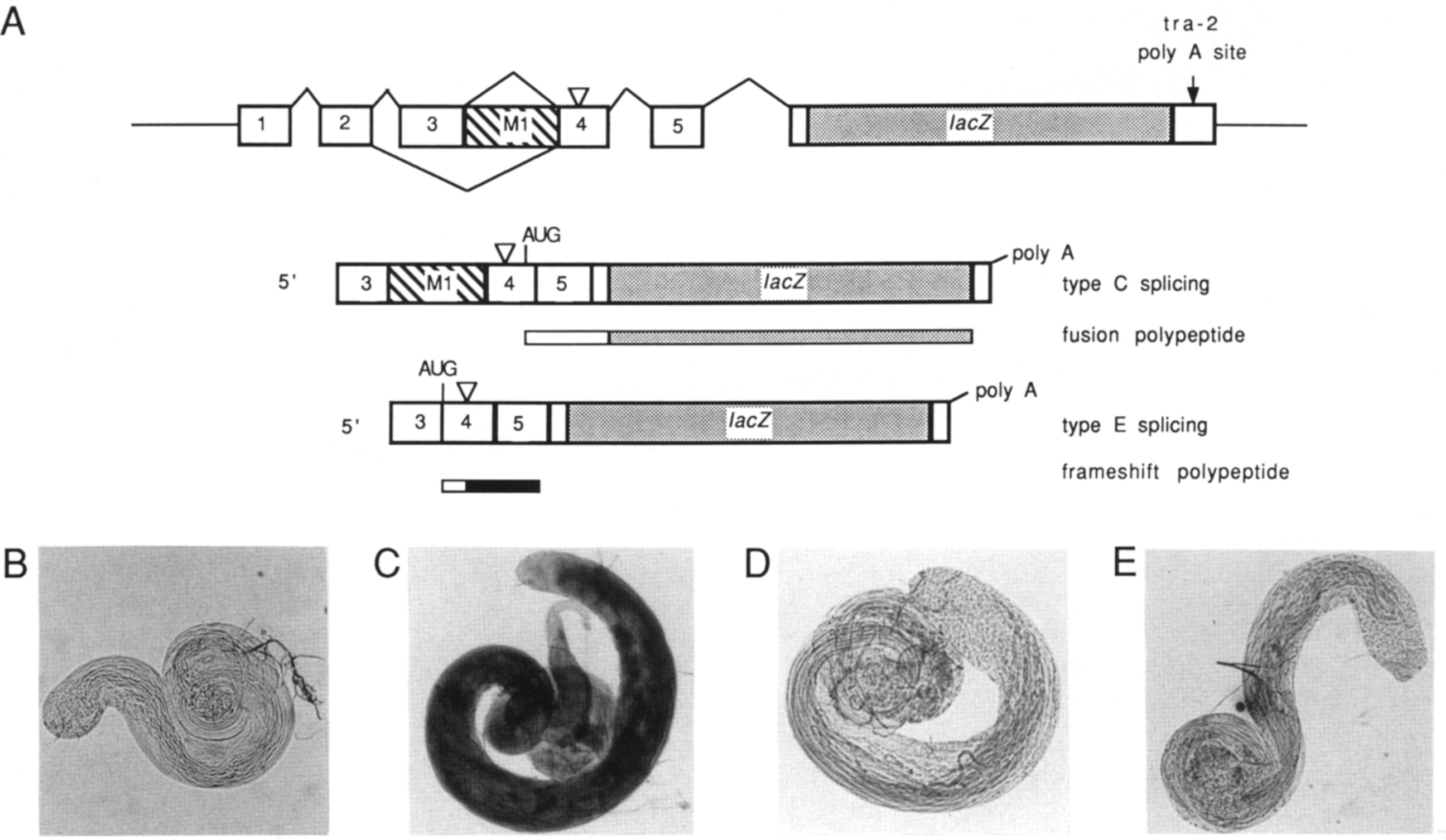

Figure 5. Trans-regulation of a tra-2/lac $Z$ fusion gene by the endogenous tra-2 gene. $(A)$ The structure of the fusion gene contained in pCZPORF3 is shown. The structure of the fusion mRNAs expected in the male germ line and the polypeptides that would be translated from them are also shown. The triangle above exon 4 indicates the position of the 7 -nucleotide insertion mutation introduced in vitro. In all cases, the shaded region indicates $l a c Z$ sequences. All other sequences derive from the tra-2 gene. The dark region of the polypeptide translated from type $\mathrm{E}$ fusion RNA corresponds to sequences that are expected to be read in the wrong reading frame. Also shown is X-gal staining of testes from $B, a+/ t r a-2^{B}$ male that does not carry the reporter gene; $C$, a $+/$ tra- $2^{B}$ male with one dose of the reporter gene; $D$, a tra $-2^{B} / \operatorname{tra}-2^{B}$ male with one dose of the reporter gene; and $E$, a $\operatorname{tra}-2^{1} /$ tra $-2^{1}$ male with one dose of the reporter gene. 
predicted tra-2 type $\mathrm{C}$ product followed by lacZ sequences. This tra-2/lac $Z$ fusion polypeptide is diagramed in Figure 5A.

The reporter gene in pCZPORF3 was introduced into the Drosophila genome by P element-mediated transformation (Rubin and Spradling 1982; Spradling and Rubin 1982), and five independent lines were obtained. The appropriate crosses were then performed to obtain individuals carrying both the reporter gene and various combinations of tra-2 alleles.

Because we wished to test the expression of this reporter gene in the absence of any tra-2 function it was important to verify that the reporter gene itself provides no $t r a-2^{+}$activity. Therefore, the ability of the reporter gene to complement known tra-2 mutants was examined. As expected, we found that the presence of the reporter gene had no discernible effect on the defects in somatic sexual differentiation and male fertility normally associated with $\operatorname{tra}-2^{1} / \operatorname{tra}-2^{1}$ and $\operatorname{tra}-2^{B} / \operatorname{tra}-2^{B}$ individuals (data not shown).

Having confirmed that it has no $\mathrm{tra}-2^{+}$activity, we examined the expression of the reporter gene in the presence of wild-type and mutant endogenous tra-2 alleles. As shown in Figure 5C, males carrying the reporter gene and a wild-type tra-2 gene $\left(\right.$ tra $\left.-2^{B}\right)+\mid$ show strong X-gal staining within their testes and therefore express the fusion protein. When males carrying the reporter gene and lacking a functional tra-2 gene $\left(t r a-2^{B} / \operatorname{tra}-2^{B}\right.$ and $t r a-2^{1} /$ tra- $2^{1}$; Fig. 5D,E) were similarly treated, virtually no $\mathrm{X}$ gal staining of testes was obtained /cf. testes in Fig. 5,D and $E$, with the control in Fig. 5B, which lacks the reporter gene). We interpret this to mean that the accumulation of transcripts containing the $\mathrm{Ml}$ intron depends on the presence of a functional tra-2 gene provided in trans. This strongly supports the idea that tra-2 products play a critical role in regulating the expression of type $C$ RNA and that the absence of type C RNA from mutant strains results from the absence of functional tra-2 polypeptides. This conclusion is confirmed below in nuclease protection experiments that more directly examine trans regulation of the expression of type C RNA molecules by tra- 2 products.

Products encoded by either type $A, B$, or E transcripts are needed for regulation of type C RNA accumulation

The accumulation of type $C$ transcripts might be regulated by polypeptides encoded by the other tra-2 transcripts (types A, B, or E). Alternatively, a basal level of the polypeptide encoded by type C RNA itself might act to regulate its own synthesis positively. To determine which of these possibilities is correct we introduced the same frameshift mutation present in pCZPORF3 into an otherwise intact copy of the tra-2 gene. As outlined above, only the polypeptide encoded by type $\mathrm{C}$ transcripts can potentially be translated in the presence of this frameshift mutation. A 4-kb EcoRI fragment containing this altered gene and all flanking sequences necessary for normal tra-2 functioning (Goralski et al. 1989) was then inserted into the P-element transformation vector pCasPeR to generate the plasmid pCSPORF3. We then introduced the $P$ element on this plasmid into the Drosophila genome and tested the ability of the frameshifted tra-2 gene to regulate type C RNA expression.

To distinguish type $C$ transcripts expressed by the tra2 gene on pCSPORF3 from those expressed by the endogenous tra-2 genes we performed nuclease protection experiments that exploited the presence of the 7-nucleotide insertion in pCSPORF3 (see Fig. 6A). When an antisense probe containing the 7-nucleotide insertion anneals with transcripts from the endogenous tra-2 genes, the inserted nucleotides are expected to "loop out" of the hybrid and be cleaved during nuclease digestion. When the same probe anneals with transcripts deriving from
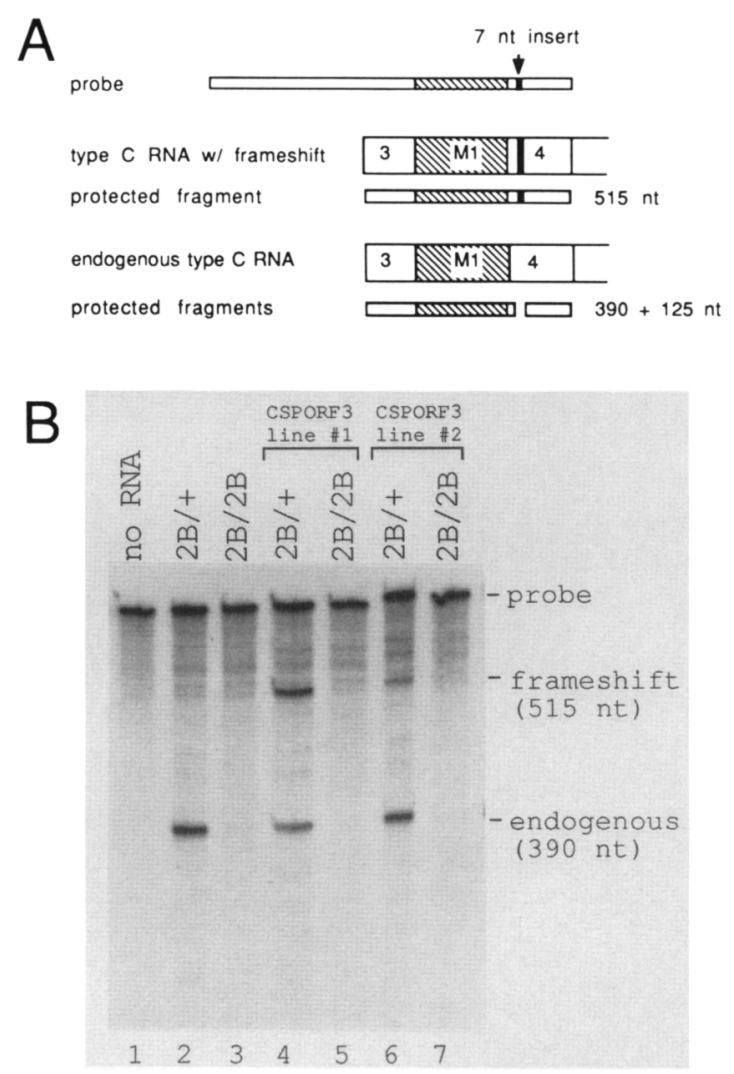

Figure 6. Failure of pCSPORF 3 to autoregulate $M 1$ splicing. (A) Shown is the strategy for distinguishing between type $C$ transcripts deriving from the tra-2 gene on PCSPORF3 and those deriving from the endogenous tra-2 gene. The position of the frameshift mutation is indicated in all cases by the dark region in exon 4. Nuclease protection products from the two expected classes of type $\mathrm{C}$ transcripts are shown. Other types of tra-2 RNAs (types A, B, E) yield only lower molecular weight fragments (31-188 nucleotides) with the probe used. $(B)$ Products from nuclease protection reactions are shown after electrophoresis on a denaturing polyacrylamide gel. Before nuclease digestion the probe was hybridized with either no RNA (lane 1) or poly $(\mathrm{A})^{+}$RNA from $+/$tra $-2^{B}$ males (lane 2 ), $t$ tra $-2^{B} /$ tra $-2^{B}$ males (lane 3 ) $+/$ tra $-2^{B}$ males carrying the $P$ element (lanes 4 and 6 ), or $\operatorname{tra}-2^{B} /$ tra $-2^{B}$ males carrying the P element (lanes 5 and 7 ). Lines 1 (lanes 4 and 5) and 2 (lanes 6 and 7) were derived from independent $\mathrm{G}_{0}$ injectees. 
the tra-2 gene on pCSPORF3, however, a perfect hybrid is expected. As a result, type $C$ transcripts deriving from pCSPORF3 should protect a 515-nucleotide fragment, whereas type $C$ transcripts originating from the endogenous tra-2 gene should yield two fragments of 390 and 125 nucleotides.

Five independent lines transformed with pCSPORF3 were analyzed for their expression of type $C$ transcripts. All lines yielded similar results. An experiment involving two such lines is shown in Figure 6B. As expected when RNA from males without pCSPORF3 was analyzed, a 390-nucleotide fragment indicative of the endogenous type $C$ transcripts was protected but not a 515 nucleotide fragment (Fig. 6B, lane 2; the 125-nucleotide protected fragment comigrates with protected fragments from somatic tra-2 transcripts and is not shown). In males carrying the tra-2 gene from pCSPORF3 both the frameshift-containing (515-nucleotide protected fragment) and endogenous type $C$ transcripts (390-nucleotide protected fragment) are expressed when a wild-type endogenous tra-2 gene is present (Fig. 6B, lanes 4 and 6). In males without a wild-type tra-2 gene $\left(\operatorname{tra}-2^{B} / \operatorname{tra}-2^{B}\right)$, however, neither type $C$ transcripts from pCSPORF3 nor the endogenous gene were detected (Fig. 6B, lanes 5 and 7). These results indicate that the tra-2 gene on pCSPORF3 is incapable of autonomously supporting the accumulation of type C RNA. Thus, one of the polypeptides encoded by the type A, B, or E mRNAs (those disrupted by the frameshift mutation) must normally play an essential role in the tra-2 autoregulatory function. Although this result argues that accumulation of type $\mathrm{C}$ RNA is not positively autoregulated by the type C polypeptide alone, it does not rule out the possibility that the type $\mathrm{C}$ polypeptide functions in combination with other tra-2 polypeptides to regulate the accumulation of type C RNA.

Given the fact that sequences coding for type A, B, and E products are disrupted in the tra-2 gene on pCSPORF3 and the above observation that this gene is incapable of autonomously expressing substantial amounts of type $\mathrm{C}$ products, one might expect that it would not provide any of the $\mathrm{tra}-2^{+}$functions needed for male fertility. Indeed, when tested, we found that pCSPORF3-bearing tra- $2^{B}$ / tra $-2^{B}$ males from each of the transformed lines were sterile (data not shown).

Do tra-2 products autoregulate the expression of male germ line transcripts by repressing RNA splicing!

Although the above data establish that tra-2 polypeptides play an important role in the accumulation of type $C$ transcripts, they do not distinguish whether this is accomplished by repressing the splicing of the $M 1$ intron or by repressing the degradation type C RNA. To distinguish between these possibilities we destroyed the splice sites flanking the $\mathrm{M} 1$ intron and examined the ability of type $C$ transcripts to accumulate in the absence of a functional tra-2 gene. If tra-2 products normally act to stabilize type C RNA, we would expect the disruption of splice site sequences to have no effect on this process.
Consequently, even type $C$ transcripts that have nonfunctional splice sites would be degraded in the absence of functional tra-2 products. If, on the other hand, tra-2 products normally regulate the accumulation of type $\mathrm{C}$ transcripts by repressing splicing of the $M 1$ intron, we would expect the disruption of these splice sites to alleviate the need for splicing repression by tra-2 products. In this case, type $C$ transcripts with nonfunctional splice sites should accumulate whether or not functional tra-2 polypeptides are present.

As shown in Figure 7A site-directed mutagenesis was used to change nucleotides at highly conserved positions in both the donor and the acceptor sites flanking the Ml
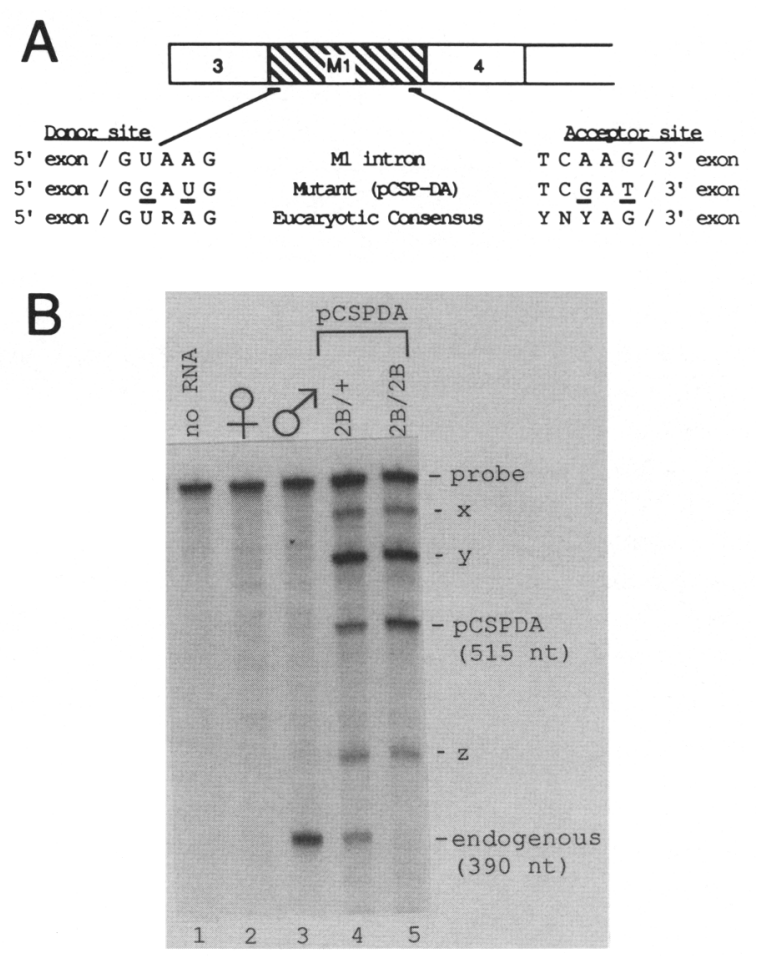

Figure 7. Effect of donor and acceptor site mutations on accumulation of type C RNA. (A) Mutations introduced at splice junction sites in PCSPDA are shown in relation to the eukaryotic consensus sequences (Green 1986). Altered nucleotides are underlined. Mutations were made in the canonical dinucleotides near both the donor site (GU) and the acceptor site (AG) in the M1 intron. $(B)$ Nuclease protection reactions were performed by using the same strategy as shown in Fig. 6A. Before nuclease digestion the probe was hybridized with either no RNA (lane 1), or poly(A) ${ }^{+}$RNA from wild-type females (lane 2), wild-type males (lane 3 ), $+/$ tra $-2^{B}$ males carrying the $P$ element from pCSPDA (lane 4), or tra $-2^{B} /$ tra $-2^{B}$ males carrying the $P$ element (lane 5). Bands corresponding to type $C$ transcripts originating from both pCSPDA and the endogenous tra-2 genes are indicated. Bands $x, y$ and $z$ appear only in lines bearing the $P$ element. Band $x$ corresponds in size to a transcript initiating at the upstream transcription start site (used in somatic and female germ-line tissues) that contains both the $M 1$ intron and the second intron. Band $y$ derives from somatic transcripts containing the Ml intron alone. Band $z$ is of unknown origin but probably results from use of cryptic splice sites. 
intron so that they differed from the eukaryotic consensus sequence (Green 1986). As with pCSPORF3, a 4-kb EcoRI fragment bearing these mutations was inserted into pCaSpeR. The resultant plasmid, pCSPDA, also contains the same frameshift mutation that was used in pCSPORF3. The presence of this mutation allows the transcripts with altered splice sites to be distinguished from endogenous transcripts in nuclease protection assays and ensures that tra-2 transcripts containing the altered splice sites do not themselves encode products with autoregulatory activity. The P element in pCSPDA was then introduced into the Drosophila genome and a number of independent transformed lines were identified. Analysis of the expression of type $C$ transcripts from two such lines yielded identical results; one of these is shown in Figure 7B. As expected from previous experiments, the accumulation of type $\mathrm{C}$ transcripts originating from the endogenous tra-2 gene was dependent on the presence of functional tra-2 products. This is demonstrated by the protection of a 390-nucleotide fragment by RNA from $t r a-2^{B} /+$ males (Fig. 7B, lane 4) but not by RNA from $t r a-2^{B} /$ tra $^{-2^{B}}$ males (Fig. 7B, lane 5 ). However, accumulation of transcripts with nonfunctional splice sites from pCSPDA was not dependent on the presence of functional tra-2 products (Fig. 7B, lanes 4 and 5). Thus, mutations in the splice sites flanking the $\mathrm{Ml}$ intron disrupt the process that is normally responsible for the absence of type $C$ transcripts in tra- $2^{B}$ males. This is consistent with the idea that tra-2 products normally function to repress splicing of the $\mathrm{Ml}$ intron and argues against the idea that tra-2 products function to repress the degradation of tra-2 transcripts through a process unrelated to RNA splicing.

\section{Discussion}

The studies presented here were carried out to investigate the effects that tra-2 null point mutations have on the accumulation of specific tra-2 transcripts. These mutations appear to affect the splicing of the Ml intron from transcripts that are expressed in the male germ line. This intron is normally spliced inefficiently in the male germ line so that transcripts containing it (type C) are more prevalent than those from which it has been removed (type E). In males homozygous for the tra- $2^{B}$ mutation, type $\mathrm{C}$ transcripts fail to accumulate and type $\mathrm{E}$ transcripts are increased in amount. The effect of tra-2 mutations on type C RNA is not likely to be the result of a defect in RNA stability, as mutations in the splice sites flanking the $M 1$ intron disrupt the events responsible for the absence of Ml-containing transcripts in tra-2 mutant males. These and other results strongly suggest that null mutations in the tra-2 gene increase the efficiency of $\mathrm{Ml}$ splicing and thereby cause a dramatic decrease in the level of type $C$ transcripts.

Analysis of the transcripts expressed by reporter gene constructs in the presence and absence of a functional tra-2 gene leads us to conclude that the products of the tra-2 gene itself normally function to regulate the levels of type $\mathrm{C}$ and $\mathrm{E}$ transcripts and that this autoregulatory function is disrupted by tra-2 mutations. We believe that our results are most compatible with the idea that wildtype product(s) of the tra-2 gene normally repress splicing of the $\mathrm{Ml}$ intron resulting in an accumulation of type $\mathrm{C}$ transcripts. In the absence of repression by tra-2 products (i.e., in mutant males) the cellular splicing machinery removes the intron from virtually all tra-2 transcripts.

The experiments presented here do not address the mechanism by which $\mathrm{M} 1$ splicing is repressed by tra-2 products, but the fact that tra-2 polypeptides contain a putative sequence-specific RNA-binding site (Amrein et al. 1988; Goralski 1989) suggests that they may bind directly on tra-2 pre-mRNA or another RNA component to block the association of small nuclear ribonucleoproteins (snRNPs) or other essential splicing factors with the pre-mRNA. One interesting alternative possibility, also consistent with our current data, is that tra-2 products play a critical role in the nuclear export of type $\mathrm{C}$ transcripts in a manner analogous to the way that the $R e v$ gene of the human immunodeficiency virus acts in the export of unspliced viral transcripts /Chang and Sharp 1989; Malim et al. 1989|. If tra-2 plays such a role, the apparent repression of Ml splicing by tra-2 might be explained as a secondary effect of the rapid tra-2-facilitated transport of these molecules to the cytoplasm where they are inaccessible to splicing factors.

An interesting, and perhaps informative, parallel to autoregulation of tra-2 RNA splicing is provided by the Sex-lethal $(S x I)$ gene, which is another member of the Drosophila sex determination regulatory hierarchy. Sxl contains two RNA-binding regions (Bell 1988) like the one found in tra-2 and has recently been shown to autoregulate splicing of its transcripts (Bell et al. 1991). The fact that this autoregulatory property is shared by the only two known developmentally specific regulators of RNA splicing with such RNA-binding domains suggests that autoregulation of RNA splicing may be a common feature of such genes.

The existence of an elaborate autoregulatory mechanism that controls the splicing of tra-2 RNA in the male germ line raises the issue of what role autoregulation plays in the expression of tra-2 products during spermatogenesis. To understand this it is first necessary to establish which of the various tra-2 products is the effector of splicing repression and to determine the functions performed by the tra-2 products that are regulated by its action. The effector molecule itself is likely to be a tra-2 polypeptide, rather than a tra-2 RNA molecule, as the autoregulatory function can be disrupted by either a nonsense mutation $\left(t r a-2^{B}\right)$ or a frameshift mutation (in pCSPORF3). The $\operatorname{tr} a-2^{B}$ mutation is predicted to affect the polypeptide products encoded by all four of the major tra-2 transcripts, whereas the frameshift mutation in pCSPORF3 is predicted to affect only the polypeptides encoded by RNA types A, B, and E. Among these, type A and B RNAs are expressed in somatic tissues and in the female germ line but have not been detected in the male germ line (Mattox et al. 1990; W. Mattox, unpubl.). This leaves the polypeptide encoded by type $\mathrm{E}$ as the best 
candidate for the effector of autoregulation. Because repression of $\mathrm{Ml}$ splicing leads to lower levels of type $\mathrm{E}$ RNA, the tra-2 autoregulatory function could serve as a negative feedback mechanism for controlling the level of type E product expressed. Similar negative feedback mechanisms have been proposed to control the level of products from the yeast ribosomal protein gene L32 (Dabeva et al. 1986) and the Drosophila su $\left(w^{a}\right)$ gene $(\mathrm{Za}-$ char et al. 1987), both of which also function to autoregulate the splicing of specific introns within their own pre-mRNAs.

The possibility exists, however, that a very low level of type A and B transcripts is present in the male germ line and has escaped detection. If so, their products could play a role in repression of M1 splicing. We are currently performing a more extensive molecular genetic analysis of the roles played by each of the various tra-2 products in repression of $\mathrm{M} 1$ splicing.

The finding that a tra-2 product other than that encoded by type C RNA is required for male fertility is consistent with the recently reported findings from germ-line transformation studies using tra-2 cDNA clones transcribed from the $h s p 70$ gene promoter (Amrein et al. 1990). These studies demonstrated that the product encoded by type C RNA is alone insufficient for male fertility.

Because the type $\mathrm{C}$ transcript contains an unprocessed intron, it seems possible that this RNA remains untranslated and retained within the nucleus as are most premRNAs (Bastos and Avis 1977; Nevins 1979). However, type C RNA contains a 179 -amino-acid ORF and therefore has the potential to function as an mRNA. Experiments presented here demonstrate that when transcripts bearing the $M 1$ intron are produced from a tra-2/lacZ fusion gene (as in pCZPORF3), they are translated within the male germ line. Thus, transcripts containing this intron can be transported to the cytoplasm and function there as mRNAs. If type C RNA is translated into a polypeptide in the male germ line, repression of Ml splicing could act as a switch, reducing expression of the type E product and simultaneously increasing expression of the type $\mathrm{C}$ product.

A notable feature of the tra-2 splicing autoregulatory function is that it appears to be tissue specific. Polyadenylated tra-2 transcripts containing the $M 1$ intron are not detected in somatic tissues or in the female germ line. Although it is possible that this is somehow due to differences in the types of tra-2 polypeptides expressed in various tissues, it should be pointed out that type $B$ and E mRNAs encode the same 226-amino-acid polypeptide. Thus, the polypeptide which is the most likely candidate for the effector of autoregulation, is probably also present in tissues where splicing of the Ml intron is not repressed. Another possibility is that the splicing of the M1 intron is more readily repressed in transcripts initiating at the male germ-line start site than in transcripts initiating at the upstream start site that is used in other tissues. However, we have recently found that the M1 intron is spliced efficiently when transcripts initiating at the male germ-line start site are expressed ectopically in somatic tissues or in the female germ line (W. Mattox, unpubl.). This argues that some trans-acting factor required for M1 splicing repression is present only in the male germ line or that an antirepressor is present in other tissues. Such a factor may have a role similar to that of the transformer gene product in controlling tra-2 activity in somatic tissues. tra-2 polypeptides are expressed in both the male and female soma but are able to affect $d s x$ RNA splicing only in the presence of the female-specific transformer product (McKeown et al. 1988; Mattox et al. 1990).

What is the ultimate role of tra-2 products in the male germ line? From previous studies we know that tra-2 function is required in the male germ line for fertility (Watanabe 1975). Early stages of spermatogenesis proceed normally in males homozygous for tra-2 null mutations and apparently normal bundles of sperm tails develop, but sperm heads fail to elongate and eventually are positioned abnormally with respect to the tails (Belote and Baker 1983). Temperature-shift studies using a temperature-sensitive tra-2 allele demonstrated that tra2 function is required in the male germ line only during a discrete period around meiosis (Belote and Baker 1983). Although these observations do not define a specific role for tra-2 products in the male germ line, the fact that germ cells of tra-2 null mutants go through many steps that are characteristic of male differentiation makes it seem unlikely that tra-2 plays a direct role in the establishment of sexual identity in the male germ line. Rather, it seems more likely that tra-2 functions in a specific spermatogenic process.

Given the similarity of tra-2 products to RNA-binding proteins and the observation that tra-2 products can function to control alternative splicing decisions in both the germ line and the soma (Nagoshi et al. 1988, and this report), it seems that a likely molecular role of tra-2 products during spermatogenesis is in the control of the RNA processing or stability of transcripts from other genes. This idea is supported by the recent finding that the $\operatorname{tra}-2^{B}$ mutation affects the sex-specific alternative splicing and/or polyadenylation of transcripts that are expressed from the exuperantia $(e x u)$ gene in the male germ line (T. Hazelrigg, pers. comm.). Although the functioning of the exu gene is required for male fertility (Hazelrigg et al. 1990), it is presently unclear whether the effects of tra-2 mutations on male fertility are due to effects on exu RNA processing. Therefore, it is possible that tra-2 products function in the control of still other RNA processing reactions during spermatogenesis.

Regardless of whether or not tra-2 products have more, as yet undiscovered, functions, the results presented here, coupled with previous studies on the role played by the tra-2 gene in sexual differentiation, indicate that its polypeptides have the remarkable ability to regulate several very different types of alternative RNA-processing choices (see Fig. 8). In somatic tissues, tra-2 products function to regulate the selection of alternative $3^{\prime}$ end exons in $d s x$ pre-mRNA. In contrast, tra-2 products function in the male germ line to repress splicing of the M1 intron per se. Regulation of the exu gene appears to in- 
RNA:

Tissue:

transformer-2

male germ line

pre-mRNA
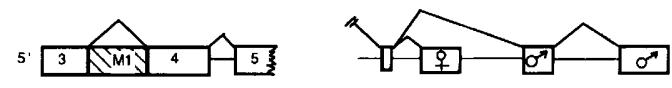

$\operatorname{tra}^{+} 2^{+}$

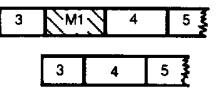

$\operatorname{tra}^{-}$

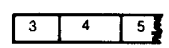

IIF" $\sigma^{\text {poly A }}$

Figure 8. The tra-2 gene regulates different kinds of alternative RNA splicing decisions. The splicing patterns of tra-2 and $d s x$ transcripts in both the presence and absence of functional tra-2 products are shown. In the case of $d s x$ RNA, the tra-2 wild-type function is required for splicing in the female-specific pattern. In the absence of this function the male-specific pattern of splicing is observed exclusively in both chromosomal male and female individuals.

volve still a different type of RNA processing choice between splicing and polyadenylation. Recent studies demonstrate that there is considerable overlap in the functional capabilities of alternative tra-2 products (Amrein et al. 1990; T.J. Goralski and B.S. Baker, unpubl.) and argue that the apparent versatility of tra-2 products cannot be explained simply by the presence of different tra-2 isoforms in different tissues. Although it remains possible that each of the tra-2 polypeptides is a highly versatile molecule capable of carrying out different processing functions when faced with different pre-mRNAs, it seems more likely that these polypeptides perform a single biochemical function that is utilized in different ways in the processing of each type of pre-mRNA. For instance, tra-2 might bind directly to a structure shared by pre-mRNA from all three genes and, in doing so, either prevent or facilitate the interaction of other splicing factors at a nearby position on the pre-mRNAs. If this were the case, it is easy to imagine that binding of tra-2 polypeptides at different positions relative to splice sites might have different effects on processing. Thus, binding of tra-2 polypeptides to a site in $d s x$ pre-mRNA might make the female-specific splice acceptor site more accessible to the splicing machinery, whereas binding of tra-2 polypeptides to tra-2 pre-mRNA might block the splicing machinery from interacting with the $M 1$ intron. In this regard, it is notable that a short sequence (ATCATTCAAC) located 57 nucleotides upstream of the M1 intron is similar to a sequence repeated six times within the $d s x$ female-specific exon $\left(T /{ }_{A}^{T} /{ }_{A} C ~ A / G\right.$ ATCAAC). Analysis of the possible binding of tra-2 polypeptides to $d s x$ and tra-2 pre-mRNAs should reveal whether this sequence is recognized by tra-2.

The ability of the tra-2 gene to participate in the control of at least three different RNA-processing decisions suggests that other splicing regulatory molecules may also control multiple splicing choices. Indeed, the $S x l$ gene, one of the few other such factors that have been identified, regulates at least two different RNA-processing decisions (Nagoshi et al. 1988; Inoue et al. 1990; Bell et al. 1991). If multiplicity of function is a common feature of such factors, numerous developmentally specific alternative splicing choices might be controlled by a relatively small group of regulatory factors.

\section{Materials and methods}

\section{Analysis of Drosophila RNA}

For RNA blot hybridization analysis $1-4 \mu \mathrm{g}$ of $\operatorname{poly}(\mathrm{A})^{+}$RNA was electrophoresed in formaldehyde-agarose gels, transferred to nylon membranes (Nytran, Schleicher \& Schuell), and hybridized as described by Rozek and Davidson (1983).

Nuclease protection experiments were performed as described previously (Mattox et al. 1990), except that hybridization reactions were performed in a volume of only $10 \mu$ l. All of the probes used were uniformly labeled, single-stranded DNAs deriving from cloned genomic tra-2 sequences.

\section{Amplification and sequence analysis of Drosophila DNA}

A small amount of genomic DNA was isolated from both homozygous tra- $-2^{B} \mathrm{cn}$ bw and mle $e^{t s} \mathrm{cn}$ bw $\left(\right.$ tra $\left.-2^{+}\right)$individuals (Davis and Davidson 1984). The mlets cn bw chromosome derives from the same screen that yielded $\operatorname{tr} a-2^{B} \mathrm{cn} b w$ and, therefore, serves as a source of the original $t r a-2^{+}$sequences (Belote and Lucchesi 1980). To amplify fragments from the tra-2 gene, polymerase chain reactions (PCRs; Saiki et al. 1988) were performed on both of these DNAs using Taq polymerase (Perkin Elmer Cetus) and several combinations of primers. From the products obtained, two restriction fragments were isolated that together cover the entire region between the male germ-line transcription start site and the carboxyl terminus of the proteincoding region. The fragments were inserted into Bluescript plasmids (Stratagene) and sequenced by using the method of Sanger et al. (1977). As a precaution against sequence changes that might occur during PCR, each of the above-described amplifications was performed in duplicate and restriction fragments from each independent reaction were cloned and sequenced. No differences between wild-type and mutant sequences were found, except for the nonsense mutation found in exon 5 that appeared in both of the duplicate clones deriving from the $t r a-2^{B}$ DNA, but not in either of the clones deriving from amplification of $t r a-2^{+}$DNA. The presence of this mutation in $t r a-2^{B}$ DNA was then confirmed by direct sequencing of PCR reaction products (without clonal isolation), using the approach described by Kusukawa et al. (1990).

\section{$X$-gal staining of testes and other tissues}

Adults were dissected in $0.7 \% \mathrm{NaCl}$ and fixed and stained with $\mathrm{X}$-gal as described by Fischer and Maniatis (1988). Staining reactions were incubated for $2-3 \mathrm{hr}$ at room temperature before mounting and photography.

\section{Construction of plasmids and in vitro mutagenesis}

The 7-nucleotide insertion contained in pCZPORF3, pCSPORF3, and pCSPDA was originally introduced into the tra-2 gene by replacing the 1.6-kb EcoRI-NaeI fragment that spans the 5 ' half of the gene with a fragment that differs only in 
that it also contains 7 nucleotides of polylinker sequences from the plasmid pTZ18R (U.S. Biochemicals) adjacent to the NaeI site. The resulting plasmid pTZ3.9A-ORF3 contains a $4.0-\mathrm{kb}$ $E c o R I$ fragment that spans the entire tra-2 transcription unit and all of the flanking sequences necessary for its expression (Goralski et al. 1989).

pCZPORF3 was generated by inserting the EcoRI-BamHI fragment of pTZ3.9A-ORF3 into pCaZPA (Mattox et al. 1990). pCSPORF3 was created by inserting the entire $4.0-\mathrm{kb}$ EcoRI fragment of pTZ3.9A-ORF3 into the EcoRI site of pCasPeR (Pirrotta 1988). To make pCSPDA, the $2.1-\mathrm{kb}$ EcoRI-SmaI fragment from pTZ3.9A-ORF3 was subcloned and subjected to site-directed mutagenesis (Zoller and Smith 1987) with oligonucleotides spanning the acceptor or donor sites of the Ml intron. Mutant-bearing clones were identified by their hybridization with the same oligonucleotides, and, after clonal isolation, the presence of the expected changes was confirmed by DNA sequence analysis. To assure that no unwanted sequence changes had occurred during this procedure the sequence of the entire transcribed portion of the EcoRI-SmaI fragment was determined after both the donor and acceptor site mutations had been introduced. A 1.6-kb BglII fragment from the mutagenized plasmid was then used to replace the analogous fragment in pTZ3.9A-ORF3, and the 4-kb EcoRI fragment from the resulting plasmid (pTZ3.9A-DA) was introduced into pCasPeR.

\section{Germ-line transformation}

P-element constructs were introduced into the Drosophila genome as described previously (Mattox et al. 1990).

\section{Acknowledgments}

We thank T. Hazelrigg for communicating results before their publication; R. Nagoshi, B. Oliver, L. Ryner, and M. Pultz for helpful discussions; and G. Bohm for preparing media and laboratory supplies. We also thank M. Garfinkel, S. Leff, P. Macdonald, and L. Ryner for providing useful comments on this manuscript. This work was supported by the California Division, American Cancer Society Fellowship S-57-89 and by a grant from the National Institutes of Health.

The publication costs of this article were defrayed in part by payment of page charges. This article must therefore be hereby marked "advertisement" in accordance with 18 USC section 1734 solely to indicate this fact.

\section{References}

Amrein, H., M. Gorman, and R. Nothiger. 1988. The sex-determining gene tra-2 of Drosophila encodes a putative RNAbinding domain. Cell 55: 1025-1035.

- 1989. Errata. Cell 58: 420.

Amrein, H., T. Maniatis, and R. Nothiger. 1990. Alternatively spliced transcripts of the sex-determining gene tra-2 of Drosophila encode functional proteins of different size. EMBO I. 9: 3619-3629.

Baker, B.S. 1989. Sex in flies: The splice of life. Nature 340: $521-524$

Baker, B.S. and K. Ridge. 1980. Sex and the single cell: On the action of major loci affecting sex determination in Drosophila melanogaster. Genetics 94: 383-423.

Baker, B.S. and M.F. Wolfner. 1988. A molecular analysis of doublesex, a bifunctional gene that controls both male and female sexual differentiation in Drosophila melanogaster. Genes \& Dev. 2: 477-489.
Bastos, R.N. and H. Avis. 1977. Globin RNA precursor molecules: Biosynthesis and processing in erythroid cells. Cell 11: 641-650.

Bell, L.R., E.M. Maine, P. Schedl, and T.W. Cline. 1988. Sexlethal a sex determination switch gene, exhibits sex-specific RNA splicing and sequence similarity to RNA-binding proteins. Cell 55: 1037-1046.

Bell, L.R., J.I. Horabin, P. Schedl, and T.W. Cline. 1991. Positive autoregulation of Sex-lethal by alternative splicing maintains the female determined state in Drosophila. Cell (in press).

Belote, J.M. and J.C. Lucchesi. 1980. Male-specific lethal mutations of Drosophila melanogaster. Genetics 96: 165-186.

Belote, J.M. and B.S. Baker. 1982. Sex determination in Drosophila melanogaster: Analysis of transformer-2, a sex transforming locus. Proc. Natl. Acad. Sci. 79: 1568-1572.

. 1983. The dual functions of a sex determination gene in Drosophila melanogaster. Dev. Biol. 95: 512-517.

- 1987. Sexual behavior: Its genetic control during development and adulthood in Drosophila melanogaster. Proc. Natl. Acad. Sci. 84: 8026-8030.

Belote, J.M., A.M. Handler, M.F. Wolfner, K.J. Livak, and B.S. Baker. 1985. Sex-specific regulation of yolk protein gene expression in Drosophila. Cell 40: 339-348.

Burtis, K. and B.S. Baker. 1989. The doublesex gene controls somatic sexual differentiation of Drosophila by producing alternatively spliced mRNAs encoding related sex-specific polypeptides. Cell 56: 997-1010.

Chang, D. and P.A. Sharp. 1989. Regulation by HIV Rev depends upon recognition of splice sites. Cell 59: 789-795.

Dabeva, M.D., M.A. Post-Beitenmiller, and J.R. Warner. 1986. Autogenous regulation of splicing of the transcript of a yeast ribosomal protein gene. Proc. Natl. Acad. Sci. 83: 5854 5857.

Davis, R.L. and N. Davidson. 1984. Isolation of the Drosophila melanogaster dunce chromosomal region and recombinational mapping of dunce sequences with restriction site polymorphisms as genetic markers. Mol. Cell. Biol. 4: 358367.

Dreyfuss, G., M.S. Swanson, and S. Pinol-Roma. 1988. Heterogeneous nuclear ribonucleoprotein particles and the pathway of mRNA formation. Trends Biochem. Sci. 13: 86-91.

Fischer, J.A. and T. Maniatis. 1988. Drosophila Adh: A promoter element expands the tissue specificity of an enhancer. Cell 53: 451-461.

Goralski, T.J., J.-E. Edström, and B.S. Baker. 1989. The sex differentiation locus transformer-2 of Drosophila encodes a polypeptide with similarity to RNA-binding proteins. Cell 56: $1011-1018$.

Green, M.R. 1986. Pre-mRNA splicing. Annu. Rev. Genet. 20: $671-708$.

Hazelrigg, T., W.T. Watkins, D. Marcey, C. Tu, M. Karow, and X. Lin. 1990. The exuperantia gene is required for Drosophila spermatogenesis as well as anteroposterior polarity of the developing oocyte, and encodes overlapping sex-specific transcripts. Genetics 126: 607-617.

Inoue, K., K. Hoshijima, H. Sakamoto, and Y. Shimura. 1990. Binding of the Drosophila Sex-lethal gene product to the alternative splice site of transformer primary transcript. $\mathrm{Na}$ ture 344: 461-463.

Kusukawa, N., T. Uemori, K. Asada, and I. Kato. 1990. Rapid and reliable protocol for direct sequencing of material amplified by the polymerase chain reaction. BioTechniques 9: 66-72.

Malim, M.H., J. Nauber, S. Le, J.V. Maizel, and B.R. Cullen. 1989. The HIV Rev trans-activator acts through a structural 
target sequence to activate nuclear export of unspliced viral mRNA. Nature 338: 254-257.

Mattaj, I.W. 1989. A binding consensus: RNA-protein interactions in splicing, snRNPs, and sex. Cell 57: 1-3.

Mattox, W., M.J. Palmer, and B.S. Baker. 1990. Alternative splicing of the sex determination gene transformer-2 is sex-specific in the germ line but not in the soma. Genes \& Dev. 4: 789-805

McKeown, M., J.M. Belote, and R.T. Boggs. 1988. Ectopic expression of the female transformer gene product leads to female differentiation of chromosomally male Drosophila. Cell 53: 887-895.

Merrill, B.M., K.L. Stone, F. Cobianchi, S.H. Wilson, and K.R. Williams. 1988. Phenylalanines that are conserved among several RNA-binding proteins form part of a nucleic acidbinding pocket in the Al heterogeneous nuclear ribonucleoprotein. J. Biol. Chem. 263: 3307-3313.

Nagoshi, R.N., M. McKeown, K.C. Burtis, J.M. Belote, and B.S. Baker. 1988. The control of alternative splicing at genes regulating sexual differentiation in D. melanogaster. Cell 53: 229-236.

Nevins, J.R. 1979. Processing of late adenovirus nuclear RNA to mRNA. J. Mol. Biol. 130: 493-506.

O'Connell, P. and M. Rosbash. 1984. Sequence, structure and codon preference of the Drosophila ribosomal protein 49 gene. Nucleic Acids Res. 12: 5495-5514.

Ota, T., A. Fukunaga, M. Kawabe, and K. Oishi. 1981. Interactions between sex transformation mutants of Drosophila melanogaster. I. Hemolymph vitellogenins and gonad morphology. Genetics 99: 429-441.

Pirrotta, V. 1988. Vectors for P-mediated transformation in Drosophila. In Vectors: A survey of molecular cloning vectors and their uses (ed. R.L. Rodriguez and D.T. Denhardt), pp. 437-456. Butterworths, Boston.

Query, C.C., R.C. Bentley, and J.D. Keene. 1989. A common RNA recognition motif identified within a defined U1 RNAbinding domain of the 70K U1 snRNP protein. Cell 57: 89101.

Rubin, G. and A. Spradling. 1982. Genetic transformation of Drosophila with transposable element vectors. Science 218: 348-353.

Rozek, C.E. and N. Davidson. 1983. Drosophila has one myosin heavy-chain gene with three developmentally regulated transcripts. Cell 32: 23-34.

Sachs, A.B., M.W. Bond, and R.D. Komberg. 1986. A single gene from yeast for both nuclear and cytoplasmic polyadenylatebinding proteins: Domain structure and expression. Cell 45: 827-835.

Saiki, R.K., D.H. Gelfand, S. Stoffel, S.J. Scharf, R. Higuchi, G.T. Horn, K.B. Mullis, and H.A. Erlich. 1988. Primer-directed enzymatic amplification of DNA with a thermostable DNA polymerase. Science 239: 487-494.

Sanger, F., S. Nicklen, and A.R. Coulson. 1977. DNA sequencing with chain-terminating inhibitors. Proc. Natl. Acad. Sci. 74: 5463-5467.

Scherly, D., W. Boelens, W.J. van Venrooij, N.A. Dathan, J. Hamm, and I.W. Mattaj. 1989. Identification of the RNAbinding segment of human Ul A protein and definition of its binding site on U1 snRNA. EMBO I. 8: 4163-4170.

Scherly, D., W. Boelens, N.A. Dathan, W.J. van Venrooij, and I.W. Mattaj. 1990. Major determinants of the specificity of interaction between small nuclear ribonucleoproteins UlA and $\mathrm{U}_{2 \mathrm{~B}^{\prime \prime}}$ and their cognate RNAs. Nature 345: 502-506.

Smith, C.W.J., J.G. Patton, and B. Nadal-Ginard. 1989. Alternative splicing in the control of gene expression. Annu. Rev. Genet. 23: 527-577.
Spradling, A. and G. Rubin. 1982. Transposition of cloned P elements into Drosophila germ line chromosomes. Science 218: 341-347.

Watanabe, T.K. 1975. A new sex-transforming gene on the second chromosome of Drosophila melanogaster. Jap. J. Genet. 50: 269-271.

Zachar, Z., T.-B. Chou, and P.M. Bingham. 1987. Evidence that a regulatory gene autoregulates splicing of its transcript. EMBO I. 6: 4105-4111.

Zoller, M.J. and M. Smith. 1987. Oligonucleotide-directed mutagenesis: A simple method using two primers and a singlestranded DNA template. Methods Enzymol. 154: 329-350. 


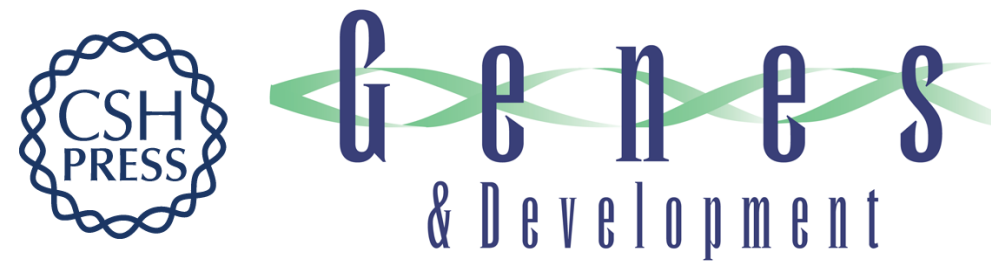

\section{Autoregulation of the splicing of transcripts from the transformer-2 gene of Drosophila.}

W Mattox and B S Baker

\section{Genes Dev. 1991, 5:}

Access the most recent version at doi:10.1101/gad.5.5.786

References This article cites 46 articles, 15 of which can be accessed free at:

http://genesdev.cshlp.org/content/5/5/786.full.html\#ref-list-1

\section{License}

Email Alerting

Service

Receive free email alerts when new articles cite this article - sign up in the box at the top right corner of the article or click here.

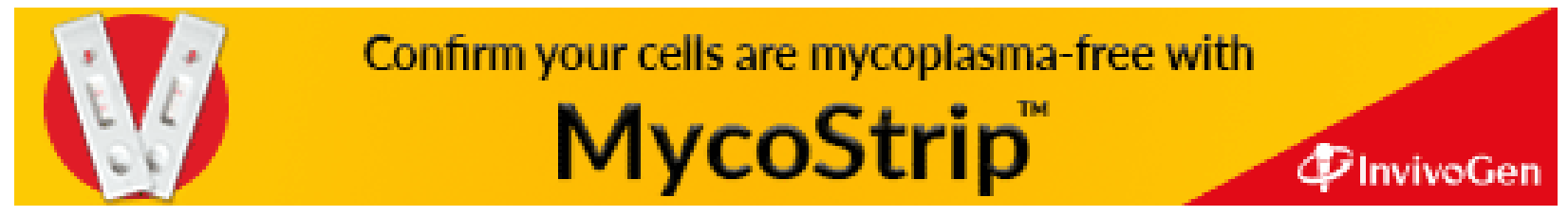

